\title{
Biomimetic Materials for the Regeneration of Extracellular Matrix in Biological Soft Tissues
}

\author{
Ei Yamamoto ${ }^{1 *}$ and Yuki Kawamura ${ }^{2}$ \\ ${ }^{I}$ Department of Biomedical Engineering, Faculty of Biology-Oriented Science and Technology, \\ ${ }^{2}$ Major of Biological System Engineering, Graduate School of Biology-Oriented Science and Technology, \\ Kindai University, 930 Nishimitani, Kinokawa, Wakayama 649-6493, Japan \\ *ei@waka.kindai.ac.jp
}

\begin{abstract}
The extensibility and elasticity of biological soft tissues such as skins, tendons, and ligaments are attributable to the quality and quantity of elastin fibers. Elastin is a representative biological polymeric fiber in the extracellular matrix of the soft tissues. The pure molecular solution of elastin is hardly extracted due to its insolubility. From this reason, many basic characteristics of elastin have not been fully elucidated not only in the area of biochemistry and molecular biology but also in that of biomechanics. The purpose of the present study was to develop biomimetic materials which include elastin ingredients for the maintenance and improvement of the skin extensibility during the wound healing. We formed the elastin-based materials whose elastic moduli were similar with those of skins. These materials were integrated with healing tissues of skin damage. These results indicate that the biomimetic materials containing elastin ingredients are useful for regenerative medicine to effectively repair skin damage.
\end{abstract}

Keywords: Biomimetic approach, Extracellular matrix, Biological soft tissue, Elastin-based material, Elastic modulus, Wound healing

\section{Introduction}

Extracellular matrix essentially comprises the structural component of biological soft tissues such as skins, tendons, ligaments, and blood vessels $[1,2]$. It consists of elastin, collagen, fibronectin, proteoglycans, and a few other glycoproteins [3]. Elastin and collagen are major biological polymeric fibers in the extracellular matrix. High purity collagen can be isolated and purified from the tissues. In contrast, the pure molecular solution of elastin is hardly extracted due to its insolubility. From this reason, many basic characteristics of elastin have not been fully elucidated not only in the area of biochemistry and molecular biology but also in that of biomechanics and biomaterial. The normal function and disease state of various tissues may be closely related to elastin components. In particular, the extensibility and elasticity of soft tissues such as skins are probably attributable to the quality and quantity of elastin fibers. However, there have not been the elastin-based materials for the regeneration of extracellular matrix in skins.
That is, few biomedical studies on elastin have been performed to repair skin damage and wound.

The sample experimentally targeted in the present study was a skin tissue as a typical example of biological soft tissues. The skin is the largest organ in the human body. As the main function of the skin, it acts like a barrier that keeps water and heat inside the body, and acts like a sensor to protect the body from various external stimuli [4]. Besides these, it plays essential roles in the control function of body temperature and the protection of internal organs. Therefore, the skin is a very important organ in life activities. There are three layers in the skin. They are the epidermis, dermis, and subcutaneous tissue from the surface to deep layer. From a mechanical point of view, it is known that the dermis mainly determines the mechanical properties of the whole skin [5]. The dermis is composed of fibroblasts and extracellular matrix that includes fibrous proteins such as collagen, elastin and proteoglycan. In particular, it is accepted that collagen and elastin are crucial 
components controlling the mechanical properties of the whole skin [6]. Collagen is closely related to the skin rigidity, elastin determines the extensibility of skin, and the mechanical properties are characterized by collagen and elastin network structure.

Our overall goal was to determine whether the external medication using biomimetic materials which include elastin ingredients is effective for the maintenance and improvement of the skin extensibility during healing process. Specifically, in the present study, we developed elastin-based materials having the same elastic moduli as those of skin tissues and studied the regenerative efficacy to repair the dermal damage using the materials.

\section{Experimental}

\subsection{Elastin purification}

Elastin ingredients were obtained from aortic bulbs of fish (black tuna) in a similar fashion as reported previously [7]. After the removal of adventitial tissues using a surgical blade, the aortic bulbs (Fig. 1) were crushed finely and agitated with a $1 \%$ sodium chloride aqueous solution and distilled water to remove blood components. Thereafter, the fat content was removed by mixing the shredded and washed bulbs with ethanol and diethyl ether. Then, insoluble elastin was obtained by vacuuming and drying. The insoluble elastin was treated with oxalic acid at $100^{\circ} \mathrm{C}$ for 1 hour, and the extract and residuum were separated using a centrifugal machine. This extracted solution was then dialyzed in cellophane tubes (21-159-2, Thermo Fisher Scientific Incorporated, Waltham, Massachusetts, U.S.A.) against distilled water. To finally obtain dry elastin powder, the solution was evaporated from the frozen state in vacuum. Figure 2 shows the purified soluble elastin which was used for the production of elastin-based materials.

\subsection{Production of elastin-based materials}

Elastin-based materials were fabricated by the same methodology as a previous report [8]. Dimethyl sulfoxide (DMSO) was used as a solvent. Elastin powders were dissolved in DMSO using a glass dish of $60 \mathrm{~mm}$ in diameter. The elastin concentration in DMSO was set at 100,150, and $200 \mathrm{mg} / \mathrm{mL}$. We then added hexamethylene diisocyanate (HMDI) to DMSO with elastin in order to increase the mechanical strength of materials. It is well known that the cytotoxicity of HMDI is lower than that of other crosslinking agents such as glutaraldehyde [9]. The concentration of HMDI in DMSO with elastin was set $15 \%$ in volume fraction. After the solution was placed in an incubator at $25{ }^{\circ} \mathrm{C}$ for 12 hours, we obtained elastin-based materials of approximately $3 \mathrm{~mm}$ in thickness and stored them in pure water for 24 hours.

\subsection{Sample preparation of skin tissues}

Six male hairless mice of 18 weeks old were used for the experiment. Their body weights were $32 \pm 4$ g. All experimental procedures using mice were approved by the Animal Experiment Committee of Faculty of Biology-Oriented Science and Technology, Kindai University. Skins were dissected from the dorsal part of each mouse. Subcutaneous layers including fat and loose connective tissues were removed from the skins using a forceps, and we prepared the skin samples composed of dermis and epidermis (Fig. 3). The thickness of the samples was approximately 800 $\mu \mathrm{m}$ in thickness.

\subsection{Indentation tests}

A conventional material tester (EZ-test, Shimadzu Corporation, Kyoto, Japan) was used for the mechanical evaluation (Fig. 4). Spherical indentation tests were conducted for the elastin-based materials and skin samples to determine their elastic moduli. The tests were performed by pressing a stiff sphere made of stainless steel into the soft specimens at room temperature in the air. The diameter of the sphere and indentation rate were $3 \mathrm{~mm}$ and $0.5 \mathrm{~mm} / \mathrm{min}$, respectively. We applied a load of $0.01 \mathrm{~N}$ to the specimens as a preload, and the force and displacement were set at zero at this condition. The push-in force was then applied to the specimens until the displacement was reached to $0.05 \mathrm{~mm}$. Applied force and displacement were recorded during the testing. From these data, we determined the elastic modulus based on Hertzian contact theory. The elastic modulus, $E$, is given as

$$
E=\frac{3 F}{4 \sqrt{r \delta^{3}}}
$$

where $F, \delta$, and $r$ denote the applied force, displacement, and sphere radius of the indenter, respectively $[10,11]$. A one-way analysis of variance (ANOVA) was used to assess the differences in the elastic modulus among the experimental groups (Elastin-based martials of 100, 150 , and $200 \mathrm{mg} / \mathrm{mL}$ in the elastin concentration and skin samples). Statistical significance was set 
at $0.1(10 \%)$.

\subsection{Skin wound healing using elastin}

Surgical treatment and experimental methods were essentially the same as those reported previously [12,13]. We used the elastin-based materials as artificial grafts to repair the skin defects. After each hairless mouse was anesthetized, a full-thickness dorsal skin was excised under aseptic condition. The skin defects were $10 \mathrm{~mm}$ square size. The experiment was conducted with two groups of animals: elastin and control groups. For the mice in the elastin group, the elastin-based material was placed on the defect, and it was covered with a commercially available adhesive plaster made of urethane film (DD-25, Aso Pharmaceutical Corporation, Kumamoto, Japan). We used the materials with the elastin concentration of 100,150 , and $200 \mathrm{mg} / \mathrm{mL}$. In contrast, the skin defects were covered only with the adhesive plaster for the mice in the control group. The motion activity and feeding behavior of the mice were checked daily throughout the experimental period of 3 weeks.

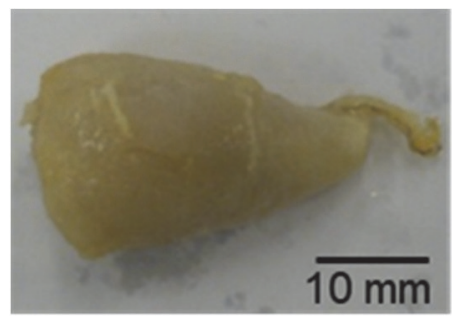

Fig. 1. An aortic bulb of black tuna. Its adventitial tissues were removed using a surgical blade.

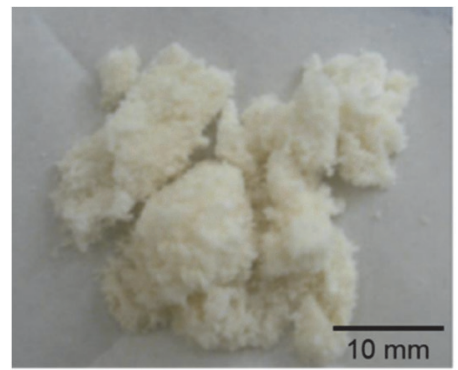

Fig. 2. Soluble elastin powders obtained from aortic bulbs. These were used for the production of elastin-based materials.

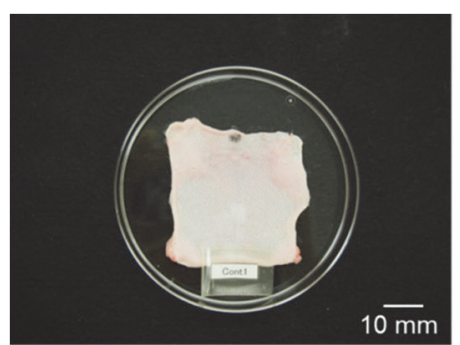

Fig. 3. A skin sample obtained from the dorsal part of hairless mouse. The test sample was composed of dermis and epidermis.

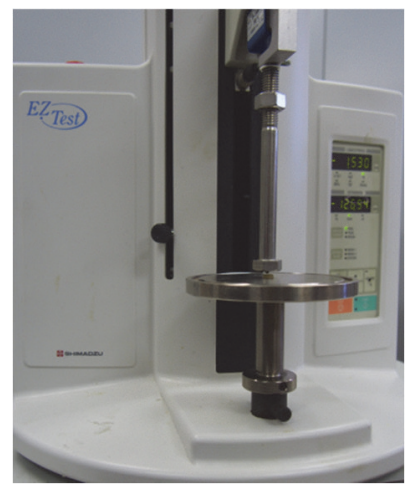

Fig. 4. A material tester used for indentation tests. The tests were conducted at room temperature in the air.

(a)

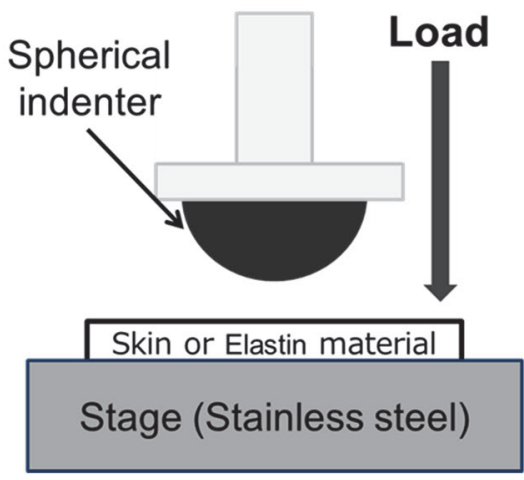

(b)

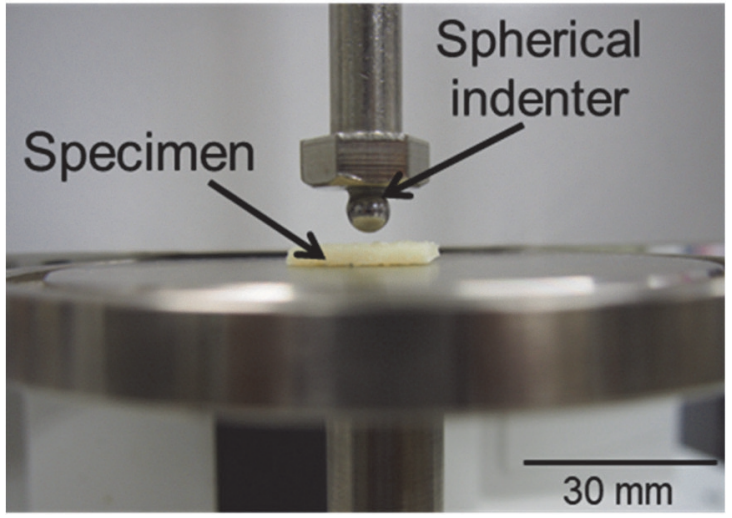

Fig. 5. A schematic diagram (a) and a photograph (b) of indentation test. A stiff sphere indenter made of stainless steel was pushed into the soft specimens. 


\section{Results and discussion}

Figure 6 shows an example of photographs of elastin-based materials. Irrespective of the elastin concentration, we could fabricate solid materials like soft hydrogels. Macroscopically, the surfaces of the materials were relatively smooth and uniform. To our knowledge, this is the first report to develop biomaterials with elastin ingredients obtained from fish aortic bulbs. In previous studies, elastin was acquired from bovine tissues. There have been zoonotic diseases between humans and cattle [14]. In contrast, it is generally known that the potential for transmission of zoonoses from fish is extremely low [15]. Therefore, the elastin-based materials fabricated in the present study can be used safely for tissue engineering applications to regenerate the extracellular matrix in such biological soft tissues as skins, blood vessels, tendons, and ligaments.

The relations between the push-in force and displacement (Fig. 7) were obtained from the spherical indentation tests. The force-displacement curves were relatively linear not only for the elastin-based materials but also for the skin samples. From these relations, we determined the elastic modulus of each specimen using Hertzian contact theory. Previously, a similar indentometoric analysis was performed to determine the elastic modulus of human skin tissues as well as artificial skin models [10]. The elastic moduli of elastin-based materials of 100,150 , and 200 $\mathrm{mg} / \mathrm{mL}$ in the elastin concentration were $200 \pm 100$, $480 \pm 233$, and $561 \pm 392 \mathrm{kPa}$ (Mean \pm S.D.), respectively, while that of skin samples was $531 \pm$ $203 \mathrm{kPa}$ (Fig. 8). The modulus of the materials at the elastin concentration of $100 \mathrm{mg} / \mathrm{mL}$ was lower than that of the skins. In contrast, there were no remarkable differences in the modulus between the materials at the elastin concentration of 150 or 200 $\mathrm{mg} / \mathrm{mL}$ and the skins indicative of the biomechanical similarity of the elastin-based materials to the skin tissues.

Figure 9 shows the scars at the skin defect at the healing period of 3 weeks. According to macroscopic observations, a deep scar remains at the skin defect in the control group. As compared with the control group, the healing of the skin defect was qualitatively fast in the elastin groups. In particular, skin wounds were more likely to heal if they are treated with the elastin-based materials of 150 or $200 \mathrm{mg} / \mathrm{mL}$ in the elastin concentration. These results indicate that skin wound healing can be promoted by the elastin-based materials that have the same elastic modulus as skin tissues. It is well known that mechanical stress can influence the skin wound healing by affecting the behavior of cells within the dermis [16]. Taken together, our findings support the concept that the mechanical properties of scaffolds are important factors to repair biological tissues in regenerative medicine. That is, the healing process of the tissues would be enhanced by an appropriate use of the biomimetic materials.

(a)

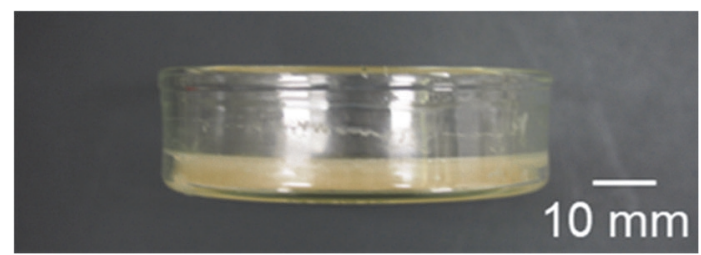

(b)

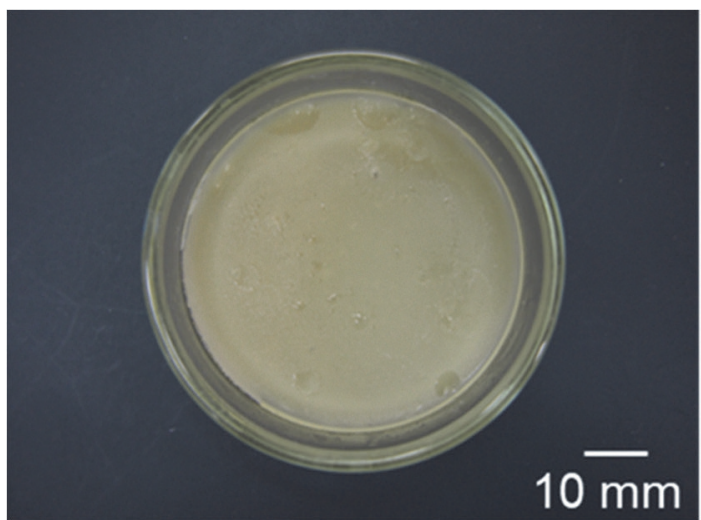

Fig. 6. Side (a) and top (b) view of an elastin-based material of $150 \mathrm{mg} / \mathrm{mL}$ in the elastin concentration. The material was fabricated in a glass dish.

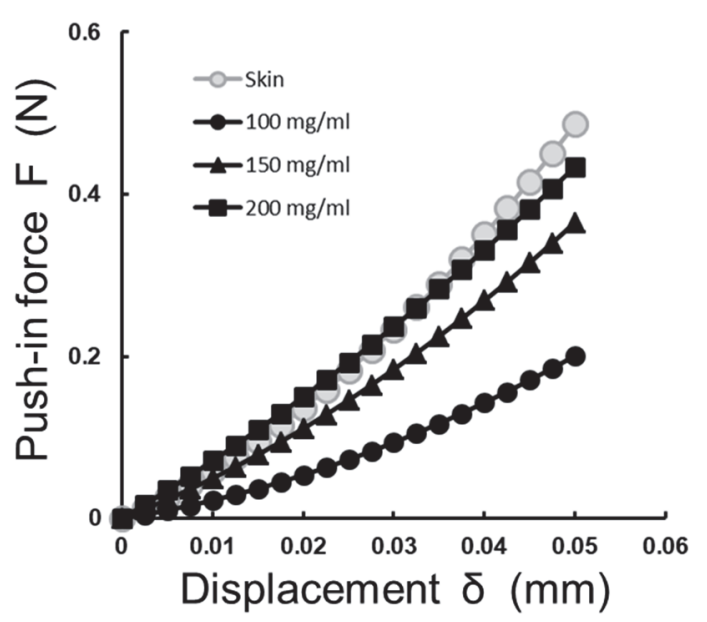

Fig. 7. Relationships between the push-in force and displacement of the elastin-based material of 100, 150, and $200 \mathrm{mg} / \mathrm{mL}$ in the elastin concentration and skin samples. These data were obtained from the indentation tests. 


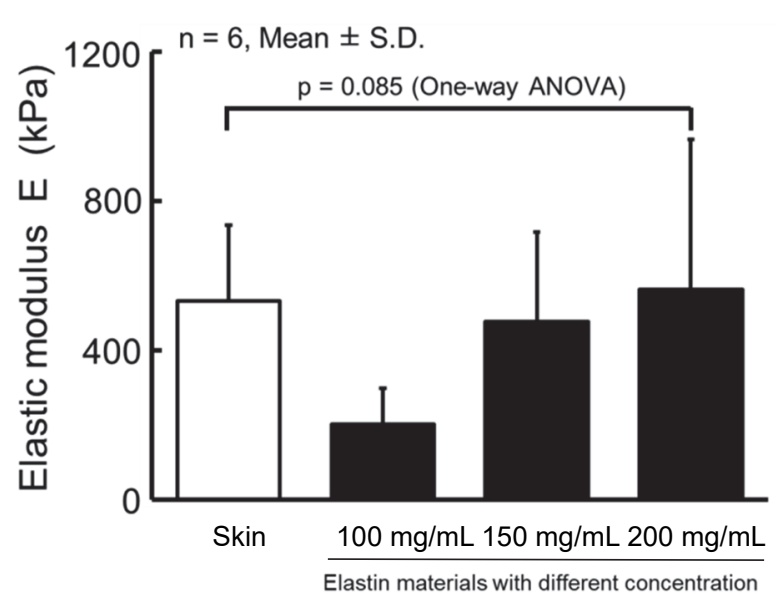

Fig. 8. Elastic modulus of the elastin-based materials of 100,150 , and $200 \mathrm{mg} / \mathrm{mL}$ in the elastin concentration and skin samples. The modulus was calculated from the push-in force and displacement of each specimen using Hertzian contact theory.

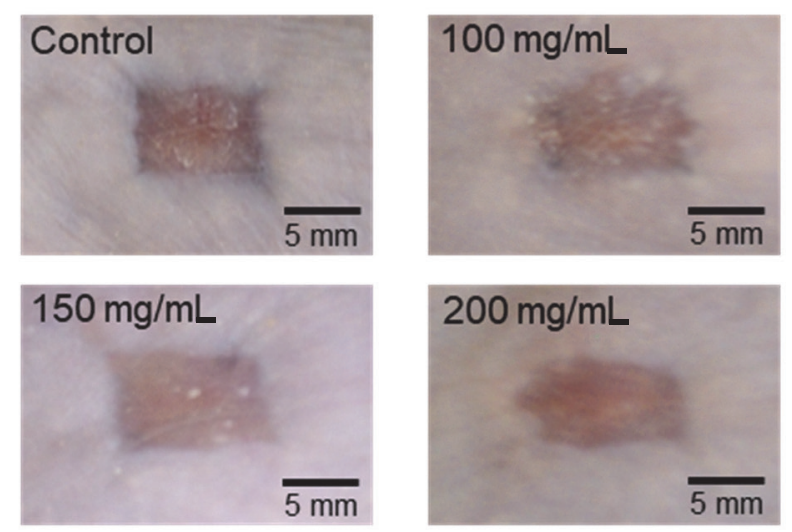

Fig. 9. Scars at the skin defects at the healing period of 3 weeks. Elastin-based materials were integrated with healing tissues of skin damage.

Collagen is the most abundant protein in the extracellular matrix of the body. Because it is easy to obtain highly soluble and pure collagen, collagen-based materials have been frequently used for tissue engineering [17,18]. In contrast, elastin is a highly insoluble protein, and thus there are relatively few applications in biomedical fields. The main strength of the present study is to fabricate bulk materials with elastin ingredients. Despite this strength, there were a few considerable limitations. Firstly, no microstructural and ultrastructural assessment was conducted for the materials in the present study. Microscopic porosity of biomaterials presumably affects the cellular adhesion and proliferation [18-20]. Secondly, we simply determined the elastic modulus of the elastin-based materials or skin tissues using Hertzian contact theory under the assumption of the linear isotropic elasticity.
However, the viscoelastic, anisotropic, and inhomogeneous characteristics would be important for polymeric hydrogels or fibrous tissues $[10,21,22]$. Thirdly, since only gross observations were performed for the scar tissues after the skin wound healing, there were no data on their histological and biochemical properties. Further studies need to be carried out in order to investigate the microstructure, viscoelasticity, and anisotropy of the elastin-based materials as well as the role of elastin in the regeneration of biological soft tissues.

\section{Conclusion}

We fabricated the elastin-based materials whose elastic moduli were similar with those of skins. These materials were integrated with healing tissues of skin damage. These results indicate that the biomimetic materials with elastin ingredients contribute the progress of regenerative medicine to effectively repair skin defects.

\section{Acknowledgements}

This work was partly supported by a Grant-in-Aid for Scientific Research (C) from Japan Society for the Promotion of Science (JSPS), Grant Numbers 15K01302 and 18K12046.

\section{References}

1. C. Frantz, K. M. Stewart, and V. M. Weaver, $J$. Cell Sci., 123 (2010) 4195.

2. A. D. Theocharis, S. S. Skandalis, C. Gialeli, and N. K. Karamanos, Adv. Drug Deliv. Rev., 97 (2016) 4.

3. J. K. Mouw, G. Ou, and V. M. Weaver, Nat. Rev. Mol. Cell Biol., 15 (2014) 771.

4. M. A. Farage, K. W. Miller, P. Elsner, and H. I. Maibach, Cutan. Ocul. Toxicol., 26 (2007) 343.

5. A. Oba and C. Edwards, Skin Res. Technol., 12 (2006) 283.

6. J. Aziz, H. Shezali, Z. Radzi, N. A. Yahya, N. H. A. Kassim, J. Czernuszka, and M. T. Rahman, Skin Pharmacol. Physiol., 29 (2016) 190.

7. S. M. Partridge, H. F. Davis, and G. S. Adair, Biochem. J., 43 (1948) 11.

8. N. Annabi, S. M. Mithieux, E. A. Boughton, A. J. Ruys, A. S. Weiss, and F. Dehghani, Biomaterials, 30 (2009) 4550.

9. M. J. van Luyn, P. B. van Wachem, L. O. Damink, P. J. Dijkstra, J. Feijen, and P. Nieuwenhuis, J. Biomed. Mater. Res., 26 (1992) 1091.

10. J. Jachowicz, R. McMullen, and D. Prettypaul, 
Skin Res. Technol., 13 (2007) 299.

11. K. Doi, M. Miki, and M. Morita, Trans. JSME, 82 (2016) 1 (in Japanese).

12. E. I. Shishatskaya, E. D. Nikolaeva, O. N. Vinogradova, and T. G. Volova, J. Mater. Sci. Mater. Med., 27 (2016) 165.

13. X. Wang, P. Wu, X. Hu, C. You, R. Guo, H. Shi, S. Guo, H. Zhou, C. Yu, Y. Zhang, and C. Han, J. Mech. Behav. Biomed. Mater, 56 (2016) 120.

14. C. J. McDaniel, D. M. Cardwell, R. B. Moeller, and G. C. Gray, Vector-Borne Zoonotic Dis., 14 (2014) 1.

15. S. Boylan, Vet. Clin. Exot. Anim., 14 (2011) 427.

16. L. A. Barnes, C. D. Marshall, T. Leavitt, M. S. $\mathrm{Hu}$, A. L. Moore, J. G. Gonzalez, M. T.
Longaker, and G. C. Gurtner, Adv. Wound Care, 7 (2018) 47.

17. J. Glowacki and S. Mizuno, Biopolymers, 89 (2008) 338.

18. C. M. Murphy, M. G. Haugh, and F. J. O'Brien, Biomaterials, 31 (2010) 461.

19. F. J. O'Brien, B. A. Harley, I. V. Yannas, and L. J. Gibson, Biomaterials, 26 (2005) 433.

20. W. K. Grier, E. M. Iyoha, and B. A. C. Harley, J. Mech. Behav. Biomed. Mater, 65 (2017) 295.

21. A. Vexler, I. Polyansky, and R. Gorodetsky, J. Invest. Dermatol., 113 (1999) 732.

22. A. N. Annaidh, K. Bruyere, M. Destrade, M. D. Gilchrist, and M. Ottenio, J. Mech. Behav. Biomed. Mater, 5 (2012) 139. 